\title{
Color variations of gravitationally lensed quasars as a tool for accretion disk size estimation
}

\author{
V. N. Shalyapin \\ Institute for Radiophysics and Electronics, Kharkov, Ukraine \\ email:vshal@ire.kharkov.ua
}

\begin{abstract}
Since the amplitude of microlensing variability depends on a source size the monitoring of gravitationally lensed quasars can produce the valuable information about the accretion disk size. Comparison of standard deviations in two wavebands yields useful constraints from quite moderate number of multicolor observations.

Existing optical VRI monitoring data for the quadruple gravitationally lensed quasar Q2237+ 0305 allow to obtain the source size ratio in two bands, V and R or V and I, as a function of size in the $\mathrm{V}$ band. Agreement with the standard accretion disk theory which predicts the dependency of a source size on wavelength $\sim \lambda^{4 / 3}$ can be achieved only if the quasar size in $\mathrm{V}$ band is comparable with the Einstein radius $\left(\simeq 10^{17} \mathrm{~cm}\right)$.
\end{abstract}

Keywords. Gravitational lensing - accretion, accretion disks - quasars: individual (Q2237+0305)

\section{Multiband monitoring of Q2237+0305}

Monitoring of microlensing variability of Q2237+0305 continues for more than 20 years, however, most of the observations have been carried out in a single band. In this study we limit ourselves by only 42 time moments when simultaneous observations in three wavebands $\mathrm{V}, \mathrm{R}$ and I are available. VR color diagram for all 4 components A-D is shown in Fig. 1a. Variations of all images on color diagrams follow along straight lines with regression slopes $0.91 \pm 0.03,0.93 \pm 0.02,0.85 \pm 0.04,0.61 \pm 0.06$ for $\mathrm{A}, \mathrm{B}, \mathrm{C}, \mathrm{D}$ components respectively in VR bands and $0.86 \pm 0.04,0.83 \pm 0.02,0.71 \pm 0.04,0.56 \pm 0.04$ in VI bands.

\section{Numerical simulations and results}

We generated magnification maps by the ray-shooting method (Kayser, Refsdal \& Stabell 1986) taking into account microlensing parameters of Q2237+ 0305A,B images the convergence $k=0.4$ and the shear $\gamma=0.4$. Observed light curves are produced by moving of a source across the magnification pattern(Mortonson, Schechter \& Wambsganss 2005). In order to estimate the ratio of two variation amplitudes and build color diagrams like Fig. 1a 42 random situated points from microlensing patterns were taken at random positions (Goicoechea, Gil-Merino, Ullan et al. 2005). Variations of two sources with radii $0.1 R_{e}\left(R_{e}\right.$ is the Einstein radius) and $0.2 R_{e}$ are correlated quite closely with the regression slope $0.91 \pm 0.03$ (Fig. 1b). 10000 Monte Carlo repetitions confirm robustness of the slope estimation as $0.92 \pm 0.03$.

In order to estimate the ratio of variation dispersions for two sources magnification maps were generated for source sizes from $0.01 R_{e}$ to $2.0 R_{e}$. The dependency of the dispersion on a source size is presented in Fig. 2a. The dispersion ratio is obtained by 

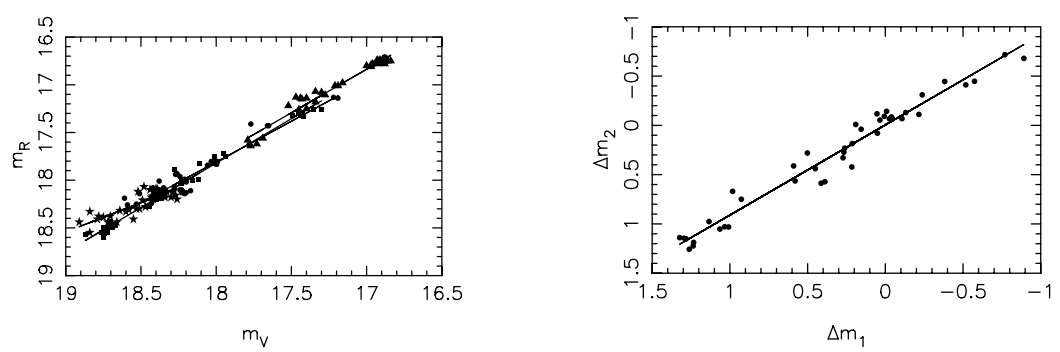

Figure 1. Left: VR color diagram of Q2237+0305. Triangle marks - A image, circles - B, squares - C, and stars - D image. Right: a sample of color variations for two sources with different radii.
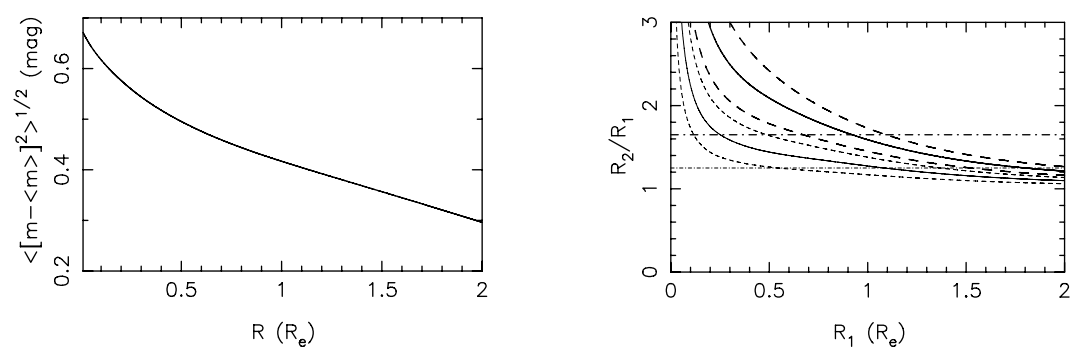

Figure 2. Left: standard deviation of microlensing fluctuations as the function of a source radius. Right: source sizes ratio for $\mathrm{R}$ and $\mathrm{V}$ bands (thin lines) and $\mathrm{I}$ and $\mathrm{V}$ bands (thick lines). Horizontal lines show the predictions from the standard accretion disk theory.

dividing two ordinate values for corresponding source sizes. This ratio cannot be presented as a function of only the source sizes ratio $R_{2} / R_{1}$ but it depends on both $R_{1}$ and $R_{2}$ in nonlinear way. So, to obtain the dispersion ratio about 0.92 as for Q2237+0305A,B values in VR diagram with a small source $R_{1}=0.01 R_{e}$ in $\mathrm{V}$ band we need choose $R_{2}=0.1 R_{e}$, i.e. in 10 times larger. For $R_{1}=0.1 R_{e}$ we need increasing of the second source only nearly in two times till $R_{2}=0.2 R_{e}$ and for very large sources an asymptotic inverse proportional of the source size (Refsdal \& Stabell 1991) is getting corrected $R_{2} / R_{1}=1 / 0.92 \sim 1.09$. Fig. 2b illustrates the above statement for two band couples VR and VI. For the last pair the dispersion ratio value 0.84 for Q2237+0305A,B was used.

It is interesting to compare the obtained results with predictions of the standard accretion disk theory which claims the power-law dependency $R(\lambda) \sim \lambda^{4 / 3}$ (Shakura \& Sunyaev 1973) (horizontal lines in Fig. 2b). Concordance of microlensing calculations with the standard accretion disk theory is reached in crossing place of the two curves (solid and dashed lines). Therefore, the RV color diagram yields the estimation of source radius in $\mathrm{V}$ band as $\sim 1.1 R_{e}$ and VI relation gives $\sim 0.9 R_{e}$, i.e. both estimations $\sim R_{e}$ that for this gravitational lens system equals $\simeq 10^{17} \mathrm{~cm}$ for solar mass microlenses.

The work was partially support by Spanish Department of Education and Science (project no. AYA2004-08243-C03-02).

\section{References}

Goicoechea, L. J., Gil-Merino, R., Ullan, A. et al. 2005, ApJ, 619, 29

Mortonson, M. J., Schechter, P. L. \& Wambsganss, J. 2005, ApJ, 628, 594

Kayser, R., Refsdal, S. \& Stabell, R. 1986, A\&A, 166, 36

Refsdal, S. \& Stabell, R. 1991, A\&A, 250, 62

Shakura, N. I. \& Sunyaev, R. A. 1973, A\&A, 24, 337 